\title{
Active Feedback Learning with Rich Feedback
}

\author{
Hang $\mathrm{Yu}^{*}$ \\ hang.yu625917@tufts.edu \\ Tufts University \\ Medford, MA, USA
}

\author{
Elaine Schaertl Short \\ elaine.short@tufts.edu \\ Tufts University \\ Medford, MA, USA
}

\begin{abstract}
In this paper, we proposed rich feedback which contains multiple types of feedback to allow human teachers to provide a variety of useful information to the learning agent and modified Policy Shaping to accumulate the effects of rich feedback. Then we designed the ALPHA framework to actively request rich feedback and further developed it to use Deep Learning. The experimental results showed ALPHA with rich feedback can greatly improve learning and quickly lead the learning agent to the optimal solution.
\end{abstract}

\section{CCS CONCEPTS}

- Computer systems organization $\rightarrow$ Robotics; • Computing methodologies $\rightarrow$ Reinforcement learning.

\section{KEYWORDS}

Human-Robot Interaction, Interactive Reinforcement Learning, Deep Learning, Rich Feedback

\section{ACM Reference Format:}

Hang Yu and Elaine Schaertl Short. 2021. Active Feedback Learning with Rich Feedback. In Companion of the 2021 ACM/IEEE International Conference on Human-Robot Interaction (HRI '21 Companion), March 8-11, 2021, Boulder, CO, USA. ACM, New York, NY, USA, 4 pages. https://doi.org/10.1145/3434074. 3447207

\section{INTRODUCTION}

Reinforcement learning is an effective technique for enabling robots and other agents to adapt their behavior to human needs and to learn to perform useful tasks[1, 2]. Recent work has used human feedback to augment these technologies, and Interactive Reinforcement Learning algorithms have shown impressive results in openAI Gym environments [3, 4] and robotic controlling problems[5, 6].

Remarkably, humans and other animals seem to solve tasks through a harmonious combination of reinforcement learning and hierarchical sensory processing systems [7, 8]. However, most methods, such as preference learning and reward learning $[3,4,9]$, are only able to effectively simulate human reinforcements or preferences. The sample complexity of these algorithms is typically high, which limits their use on real-world systems[10]. Moreover, work using human feedback often assumes that environment rewards are low-quality or unavailable and thus do not incorporate

Permission to make digital or hard copies of all or part of this work for personal or classroom use is granted without fee provided that copies are not made or distributed for profit or commercial advantage and that copies bear this notice and the full citation on the first page. Copyrights for components of this work owned by others than ACM must be honored. Abstracting with credit is permitted. To copy otherwise, or republish, to post on servers or to redistribute to lists, requires prior specific permission and/or a fee. Request permissions from permissions@acm.org.

HRI '21 Companion, March 8-11, 2021, Boulder, CO, USA

(C) 2021 Association for Computing Machinery.

ACM ISBN 978-1-4503-8290-8/21/03 . \$ \$15.00

https://doi.org/10.1145/3434074.3447207 environmental rewards. Model-based RL, on the other hand, aims to learn a model of the system, and then optimize a policy given this model. This approach has the benefit of reducing sample complexity, but the scope of the policy is limited by assumptions made in the model. In this paper, we seek to integrate human feedback with environmental rewards in reinforcement learning, especially with tasks that would trap learning agents in local optimal without human feedback, tasks that a human cannot solve perfectly, and reinforcement learning algorithms in the high dimensional state space. We propose a framework, Active Learning Plus Human Assessments(ALPHA), which allows a human teacher intuitively choose one or more pieces of distinct feedback to maximize the information gain in one interaction and enables the learning agent to active requests a certain type of feedback when doing so will accelerate learning. We include four types of feedback: demonstrations, predictions, evaluations, and flags, which are the most popular types of feedback used in most recent research. The learning agent recommends a feedback type to the annotator while the influence of the current state or trajectory is highly based on three features: Discrepancy, Leverage, and Similarity, which are proposed to measure the certainty of the decision-making. We test ALPHA in a stacking task and compare it with Q-Learning and Policy Shaping. The results show that ALPHA receives $69.0 \%$ more rewards than Q-Learning and $13.2 \%$ more rewards than Policy Shaping.

\section{RELATED WORK}

Numerous studies have addressed problems in learning from feedback in Human-Robot Interaction (HRI). There is also a large body of work on using human feedback to accelerate learning.

Many methods focus on learning from human feedback or demonstrations. Two representative methods are Policy Shaping [11, 12] and TAMER[13]. In Policy Shaping, people are allowed to give binary feedback on robots' behaviors to indicate whether it is correct. If an action receives more positive feedback, the probability of this action being chosen increases. In TAMER, humans give out scaled feedback to grade the robot's action, which is treated more or less as a reward. Note that TAMER is sensitive to early errors: an incorrect action might receive a higher reward than a correct action early in the learning process and take significant time to correct. Human feedback is also widely used in Deep Reinforcement Learning. K. Subramanian etc.[3] and B. Ibarz etc.[4] propose to use a deep neural network to learn objective rewards from human preferences and demonstrations when environmental rewards are absent.

Effectively utilizing information from human teachers is another focus in learning from human feedback. One effective method is Deep Q-Learning from Demonstration (DQfD)[9]. In DQfD, all demonstrations are permanently stored in a memory buffer and assigned a higher sampling priority than transactions that are not from demonstration. Based on the estimation of the current state 
and all past paths, P. F. Christiano etc.[14] proposed an active learning method to actively request feedback from human annotators.

\subsection{Contributions}

Our work is different from prior work in two aspects. One is we enriched the types of feedback to increase the information gain. For instance, human teachers can evaluate the last trajectory while predicting the next move for the learning agent. By combining evaluation and prediction, the quality of feedback improved since human teachers are capable of undoing their inaccurate prediction using evaluations and could even learn to give feedback from this process. Furthermore, we propose a corresponding active learning framework that automatically requests suitable feedback once the learning agent is not confident about the current state, and that also allows human teachers to actively provide rich feedback at any time point. Moreover, we also updated the formulas of the Policy Shaping to facilitate its use with Deep Learning and proposed a weight decay mechanism to balance environment rewards and human feedback that improves the final performance of deep policy shaping.

\section{METHODOLOGY}

This work builds on Interactive Reinforcement Learning (Interactive $\mathrm{RL})$ : ALPHA uses rich feedback to maximize information gain and actively requests feedback from human teachers. The Interactive $\mathrm{RL}$ algorithms we used as the basis of our work are Q-Learning and DDQN $[1,15]$ with Policy Shaping[11, 12].

\subsection{Rich Feedback}

In this work, we want to maximize the information gain in the interaction process and improve the quality of human feedback. In prior work, researchers tended to use one or two kinds of feedback, which is insufficient to express human's abstract intentions about the task. Thus, we use rich feedback to enhance learning by trying to include as much information as possible and improving the quality of feedback by the using combining different types of feedback. The rich feedback contains four distinct pieces of feedback, which are demonstrations, predictions, evaluations, and flags.

(1) Demonstration: the annotator demonstrates the following agent steps for the learning agent. Each action receives positive feedback with a value of 1 .

(2) Prediction: the annotator predicts one or more possible correct/incorrect actions for the current state. Each action receives corresponding feedback with a value in range [-2,2]

(3) Evaluation: the annotator evaluates the most recent actions Each action receives corresponding feedback with a value in the range [-3,3], which means the effects of the prediction can be undone by the evaluation.

(4) Flag: the annotator flags a state as a key state. Each action that leads to this state automatically receives positive feedback with a value of 1 or negative feedback with a value of -1 .

The three types of feedback have different weights which are set by the degree of difficulty. Giving a demonstration is the hardest and least accurate. Predicting the next move is also harder than evaluating since participants can evaluate actions by observing the consequences of actions. The value of rich feedback is calculated based on:

$$
f(s, a)=\tanh (f+\operatorname{arctanh} f(s, a))
$$

where $f(s, a)$ is accumulated feedback, $s$ is the state, and $a$ is the action. The probability of taking any action $a$ based on received feedback under the state $s$ is:

$$
P_{f}(s, a)=\frac{\beta^{f_{s, a}}}{\beta f_{s, a}+(1-\beta)^{f_{s, a}}}
$$

Where $\beta$ is the trust degree. The probability of taking any action $a$ given the learned policy in Q-Learning is:

$$
P_{Q}(s, a)=\frac{e^{\frac{Q(s, a)}{\tau}}}{\sum_{a^{\prime} \in A} e^{\frac{Q\left(s, a^{\prime}\right)}{\tau}}}
$$

The learning agent accomplishes the goal by seeking a $\hat{H}$, the action choosing policy, to maximize $P_{f}$ and $P_{Q}$ :

$$
\hat{H}=\underset{a \in A}{\operatorname{argmax}}\left[\lambda P_{f_{a}} \times P_{Q_{a}}\right]
$$

\subsection{Active Learning Strategy}

In this section, we describe an approach to identify the certainty in the interactive reinforcement learning process using statistical properties relevant to the learning states. We outline properties of different cases and actively solicit interaction to drive the agent's exploration or correct the agent's mistakes. During the learning process, the information that a learning agent can obtain is mainly the current state and recent trajectories. Thus, we propose that the Learning agent should actively ask feedback from participants based on its estimations of:

(1) The most recent Trajectory: If the most recent trajectory is an unfamiliar one, the learning agent should ask for an Evaluation to eliminate uncertainty for future learning.

(2) Recent Trajectories. If recent trajectories are similar or repeated, the learning agent should ask for an evaluation or a demonstration to avoid reward hacking.

(3) Current State: If the actions that could be chosen in the current state have similar weights, which means the learning agent is uncertain about action selection a prediction or a demonstration should be requested.

Based on the above, we proposed three formal criteria for making these decisions, where each criterion describes one case.

3.2.1 Discrepancy. This measure captures the observation that the learning agent is unable to behave appropriately thus leading to errors. $A$ is the action set.

$$
\operatorname{Disc}_{s}=\frac{1}{|A|} \sum_{a \in A}\left[P_{Q_{A}} \times P_{f_{a}}-\hat{H}\right]^{2}
$$

3.2.2 Leverage. Leverage measures the consistency of $\pi^{Q}$ and $\pi^{f}$ of the most recent trajectory:

$$
L e v=\frac{1}{|S|} \sum_{t \in T}\left[\pi_{t}^{Q} \cap \pi_{t}^{f} \cap \hat{H}\right]
$$

where $S$ is the set of the most recent transactions. 
3.2.3 Similarity. Similarity represents the ability of the learning agent to reach new states:

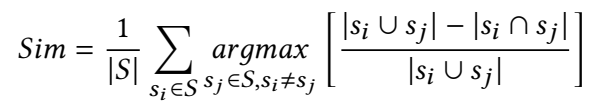

\subsection{Active Learning Plus Human Assessments (ALPHA)}

Based on the rich feedback and the active learning strategy, our Active Learning Plus Human Assessments (ALPHA) framework is described in algorithm 1:

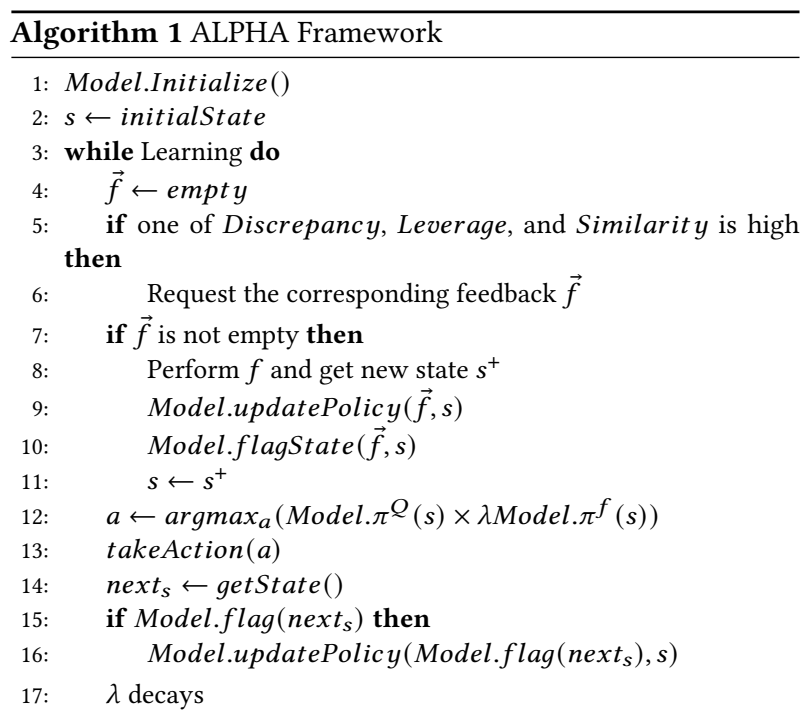

After initializing the model and getting the initial state, the learning agent starts to learn (line 1 and line 2). For each agent step, the feedback $\vec{f}$ is set to empty. Then the learning agent estimates the influence based on the current state $s$ and the recent trajectories $S$ and decides whether to request rich feedback (line 5 and line 6). If the feedback is not empty, then the model updates its feedback policy if the rich feedback contains a evaluation or a prediction, flags the current state if the rich feedback contains a flag, and updates the current state if the rich feedback contains a demonstration (line 7 to line 10). Then the agent chooses an action and moves to the move state. If the next state is a flagged state, the chosen action would receive a corresponding feedback. In the end, the agent reduces $\lambda$ to decrease the weight of feedback.

\subsection{Deep ALPHA}

To further apply ALPHA to the deep learning, we propose Deep ALPHA, which is shown in algorithm 2. The Deep ALPHA follows similar procedures as the ALPHA but has two major differences. First, deep ALPHA has a full trial demonstration and a pre-training process before the learning starts since the learning agent needs tremendous time to successfully perform for once. Second, Deep ALPHA samples transactions from the feedback buffer and updates the policy network even when there is no feedback. We also disabled the flag in the deep learning since we can not flag specific states in deep learning (attempting to do so would be either inaccurate or inefficient).

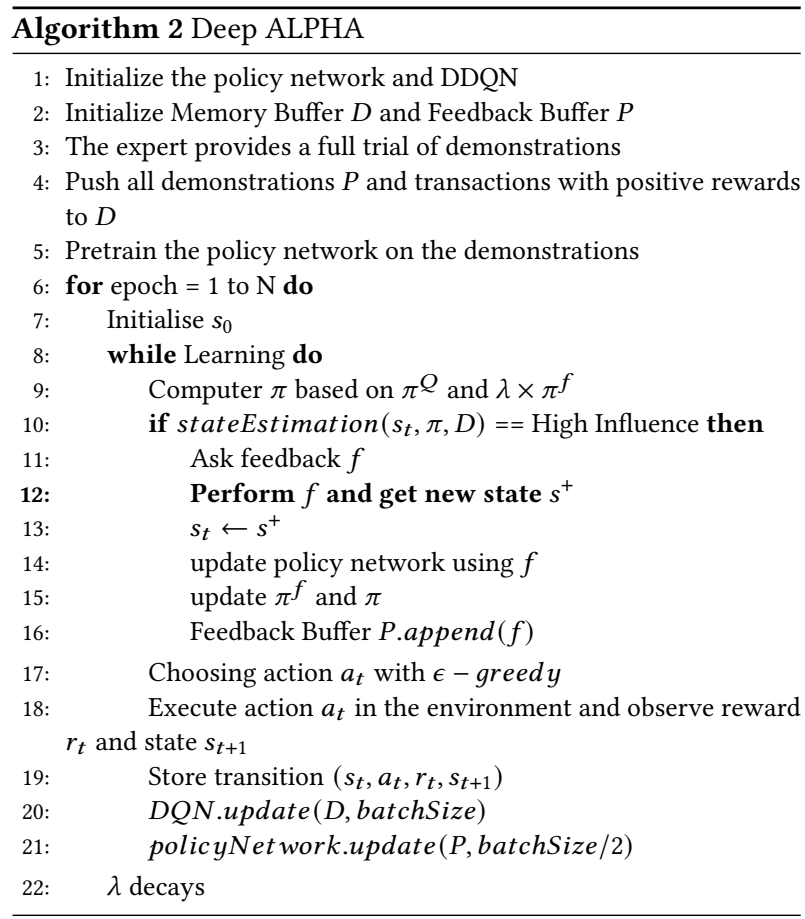

\section{EXPERIMENTS}

In this section, we constructed an oracle that always gives correct feedback and evaluated the ALPHA using simulated human feedback. We compared to two baseline methods: Q-Learning and Policy Shaping. For ALPHA and Policy Shaping, we limited the total number of feedback they can receive to 1000 . Policy Shaping received one feedback per five actions. ALPHA passively received one feedback per twenty actions and was able to actively ask for feedback. Each piece of rich feedback counts as one feedback, which means one instance of rich feedback could be counted as four feedback. Q-Learning does not receive any feedback. The task we used is a anti-greedy blocking stacking task in which the learning agent using greedy strategy would be trapped to local optimal for a long time. All results are averaged by running for 1000 trials to smooth random variations.

\subsection{Tasks}

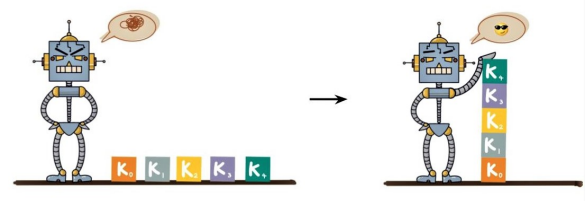

Figure 1: A block stacking task: the learning agent should learn to appropriately stack blocks to maximize the total rewards. 
In the stacking task, the learning agent needs to place five distinct blocks $\left(k_{0}, k_{1}, k_{2}, k_{3}, k_{4}\right)$ as shown in Figure 1 where each block has a value of $k_{i}=i, i=0, \ldots, 4$ and each stack from bottom to top has a ratio of $s t a c k_{j}, j=1, \ldots, 5$. For stacking a block, the learning agent would receive a reward of $k_{i} \times s t_{a c k}$ and thus is likely to get stuck at a local optimum. The maximum grade is 35 . The state set $S$ consists of all possible arrangements of blocks and the set of actions $A$ that the agent could take: Place $k_{i}$ at the top, Remove a block from the top and Restart. Each episode of training ends when the agent chooses to restart. For taking one action, the agent receives a -1 reward.

\subsection{Results}

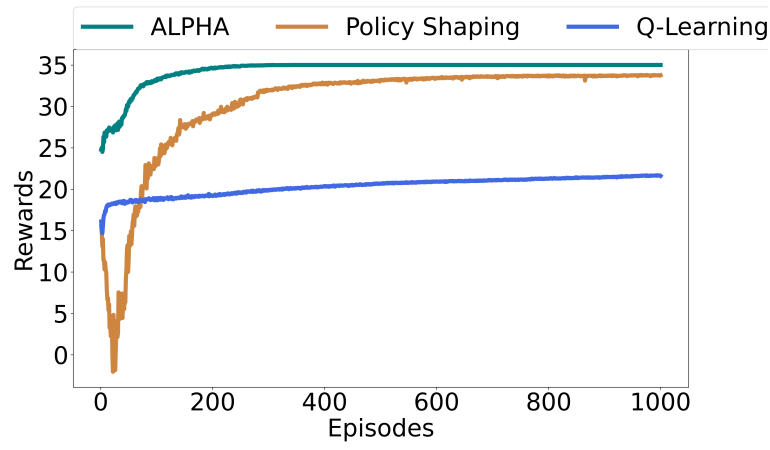

Figure 2: Experimental results for the stacking task where the ALPHA outperforms other baselines and fastest converge to the optimal solution.

We calculated the area under each curve of total rewards overall cases shown in ??. ALPHA had an average under area of 34380.7, which is $13.2 \%$ better than Policy Shaping (30373.8), and $69.0 \%$ better than Q-Learning $(20342.9)$ in the block stacking task. By using oneway $\operatorname{ANOVA}(F=3,317.17, p<0.00001)$, we found there exists significant statistical differences among these algorithms. Then, we performed a t-test with tukey's HSD test and it showed statistical differences between the ALPHA and Policy Shaping $(t=4.0069, p<$ $0.001)$, ALPHA and Q-Learning $(t=14.0377, p<0.001)$.

\section{DISCUSSION \& CONCLUSION}

The simulation results showed that the ALPHA framework received $13.2 \%$ more rewards with the same number of feedback and converges to the best solution at around 300 episodes (the Policy Shaping needs about 500 episodes).

In prior work, researchers typically only request one type of feedback from human participants. However, our work demonstrates that asking multiple types of feedback is a more effective way of interaction since humans generally not only take actions based on the current observation but also take into account prior experiences. Rich feedback is a better vehicle for high-level human instructions. Moreover, we usually improve our performance on a certain task by controlling variables and change them one by one. The flag also provides a way for human to indicate this information to the learning agent.
Although we did not test rich feedback with a human-based study, we believe that rich feedback would even gain more advantages with real humans since humans cannot always give correct feedback. Rich feedback offers a chance for them to undo their predictions and learn to give higher quality feedback. Additionally, we hypothesize that this rich feedback could more appropriately capture abstract or high-level human instructions.

In summary, we proposed rich feedback which contains multiple types of feedback to allow human teachers to provide a variety of useful information to the learning agent and modified Policy Shaping to accumulate the effects of rich feedback. Then we designed the ALPHA framework to actively request rich feedback and further developed it to use Deep Learning. The experimental results showed ALPHA with rich feedback can greatly improve learning and quickly lead the learning agent to the optimal solution.

\section{REFERENCES}

[1] S. S. Mousavi, M. Schukat, E. Howley, and P. Mannion, "Applying q ( $\lambda$ )-learning in deep reinforcement learning to play atari games," in AAMAS Adaptive Learning Agents (ALA) Workshop, 2017.

[2] X. Guo, S. Singh, H. Lee, R. L. Lewis, and X. Wang, "Deep learning for real-time atari game play using offline monte-carlo tree search planning," Advances in neural information processing systems, vol. 27, pp. 3338-3346, 2014.

[3] P. F. Christiano, J. Leike, T. Brown, M. Martic, S. Legg, and D. Amodei, "Deep reinforcement learning from human preferences," in Advances in Neural Information Processing Systems, 2017, pp. 4299-4307.

[4] B. Ibarz, J. Leike, T. Pohlen, G. Irving, S. Legg, and D. Amodei, "Reward learning from human preferences and demonstrations in atari," in Advances in neural information processing systems, 2018, pp. 8011-8023.

[5] T. P. Lillicrap, J. J. Hunt, A. Pritzel, N. Heess, T. Erez, Y. Tassa, D. Silver, and D. Wierstra, "Continuous control with deep reinforcement learning," arXiv preprint arXiv:1509.02971, 2015.

[6] J. Schulman, S. Levine, P. Abbeel, M. Jordan, and P. Moritz, "Trust region policy optimization," in International conference on machine learning, 2015, pp. 18891897.

[7] K. Fukushima and S. Miyake, "Neocognitron: A self-organizing neural network model for a mechanism of visual pattern recognition," in Competition and cooperation in neural nets. Springer, 1982, pp. 267-285.

[8] T. Serre, L. Wolf, and T. Poggio, "Object recognition with features inspired by visual cortex," in 2005 IEEE Computer Society Conference on Computer Vision and Pattern Recognition (CVPR'05), vol. 2. Ieee, 2005, pp. 994-1000.

[9] T. Hester, M. Vecerik, O. Pietquin, M. Lanctot, T. Schaul, B. Piot, D. Horgan, J. Quan, A. Sendonaris, G. Dulac-Arnold et al., "Deep q-learning from demonstrations," arXiv preprint arXiv:1704.03732, 2017.

[10] V. Mnih, K. Kavukcuoglu, D. Silver, A. A. Rusu, J. Veness, M. G. Bellemare, A. Graves, M. Riedmiller, A. K. Fidjeland, G. Ostrovski et al., "Human-level control through deep reinforcement learning," nature, vol. 518, no. 7540, pp. 529-533, 2015.

[11] T. Cederborg, I. Grover, C. L. Isbell, and A. L. Thomaz, "Policy shaping with human teachers," in Twenty-Fourth International foint Conference on Artificial Intelligence, 2015. [Online]. Available: https://www.aaai.org/ocs/index.php/IJCAI/ IJCAI15/paper/viewPaper/11412

[12] S. Griffith, K. Subramanian, J. Scholz, C. L. Isbell, and A. L. Thomaz, "Policy shaping: Integrating human feedback with reinforcement learning," in Advances in neural information processing systems, 2013, pp. 2625-2633.

[13] W. B. Knox and P. Stone, "Tamer: Training an agent manually via evaluative reinforcement," in 2008 7th IEEE International Conference on Development and Learning. IEEE, 2008, pp. 292-297.

[14] K. Subramanian, C. L. Isbell, and A. L. Thomaz, "Exploration from demonstration for interactive reinforcement learning," in Proceedings of the 2016 International Conference on Autonomous Agents amp; Multiagent Systems, ser. AAMAS '16. Richland, SC: International Foundation for Autonomous Agents and Multiagent Systems, 2016, p. 447-456.

[15] H. Van Hasselt, A. Guez, and D. Silver, "Deep reinforcement learning with double q-learning," arXiv preprint arXiv:1509.06461, 2015. 